\title{
Krise des Kapitalismus?
}

\section{Auf schwankendem Grund}

„Man muss den Kapitalismus vor den Kapitalisten schützen“, sagte schon John Maynard Keynes. Die Krise des Kapitalismus ist auch heute in aller Munde. Weltweit haben sich Protestbewegungen gebildet, die ein Umdenken in der Art und Weise des Wirtschaftens in den westlichen Industrieländern wie auch den Schwellenländern anmahnen. Am Pranger steht die scheinbar grenzenlose Verfügungsgewalt Privater über Ressourcen und Güter, die nicht mehr zum Wohle einer Gesellschaft eingesetzt wird, sondern allein der privaten Gewinnmaximierung dient, selbst wenn dies zu Lasten der Gesamtwirtschaft geht. Primär stehen dabei die Praktiken des Finanzsektors im Fokus der Angriffe. Diese verbinden sich zudem häufig mit Forderungen nach einem generell nachhaltigeren Wirtschaften, in dem ökologischen Belangen eine deutlich größere Rolle für wirtschaftliche und wirtschaftspolitische Entscheidungen zugebilligt werden soll als bisher. Derartige Forderungen finden Rückhalt bis weit in etablierte politische Parteien hinein. Die breite Unterstützung für die Occupy Bewegung macht dies anschaulich.

Gemessen an der verbalen Unterstützung fallen die realen Veränderungen und vor allem die konkrete Bereitschaft hierzu weitaus geringer aus. Auch drei Jahre nach dem G20 Gipfel von Philadelphia, auf dem eine strikte globale Regulierung des Finanzsektors ins Visier genommen wurde, halten sich die rechtlichen Umsetzungen in engen Grenzen; teilweise wird sogar die Frage gestellt, ob überhaupt die eigentlich erforderlichen Maßnahmen in Angriff genommen wurden. Zugleich wurden und werden die auf den Weg gebrachten strengeren Regulierungsvorschriften immer wieder verwässert und mit Ausnahmen versehen, so dass die ursprünglich geplante Strenge der Regulierung einer gewissen Milde gewichen ist. Das gilt für die USA wie auch die EU. Es bleibt also festzuhalten: Auch wenn die Krise des Kapitalismus in aller Munde ist, grundlegend verändert hat sich seit Beginn der Finanzkrise wenig. Lediglich unterliegen vor allem Banken nunmehr etwas härteren Beschränkungen, abgesehen davon, dass sie aus eigenem Antrieb größere Vorsorge für schwierige Zeiten getroffen haben.

Ist die Krise des Kapitalismus also eine Mode, die kommt und geht und die keine nachhaltigen Spuren hinterlässt? Dieser Frage soll im Folgenden aus einer Perspektive der ökonomischen Wissenschaft nachgegangen werden. Denn es waren Ver- 
änderungen im ökonomischen Denken, die im Laufe der Zeit jene politische Brisanz entwickelten, die zu einer weitgehenden Entfesselung der Märkte und damit privater Verfügungsgewalt beigetragen haben.

\section{Ungebremster Kapitalismus im ökonomischen Denken}

Der intellektuelle Ursprung der Krise liegt im seit den sechziger und siebziger Jahren des vorigen Jahrhunderts veränderten Denken im Mainstream der ökonomischen Wissenschaft. Seinerzeit wurden, angestoßen von Milton Friedman und weiterentwickelt von Robert Barro und vielen anderen, jene Modelle als überlegen angesehen, die die mikroökonomische Entscheidungslogik auf die gesamtwirtschaftliche Ebene übertrugen, die also mikro-fundiert waren. Nicht nur das: Eine wichtige Implikation dieser Modelle ist, dass das Wirken der einzelwirtschaftlichen Rationalität auch gesamtwirtschaftlich von Vorteil ist. Wirtschaftliche Probleme entstehen in diesem Rahmen zumeist, indem einzelwirtschaftliche Entscheidungen behindert werden. Diese starken Aussagen sind an starke Voraussetzungen geknüpft. Wichtigstes Element ist ein Preismechanismus, der hinreichend flexibel ist, alle Transaktionswünsche in einem Gleichgewichtspreisniveau zur Übereinstimmung zu bringen. Das impliziert, dass alle zu diesem Preisniveau gewünschten Transaktionen auch ausgeführt werden können; sowohl Anbieter als auch Nachfrager sind zufrieden. Dies gilt in dieser Sichtweise auch für den Arbeitsmarkt, wo flexible Lohnsätze ein Gleichgewicht herbeiführen, so dass jeder, der zu diesem Lohnsatz arbeiten will, auch Arbeit findet. Dies hat eine weitreichende Implikation: Arbeitslosigkeit tritt nur als freiwillige Arbeitslosigkeit auf, die durch - gemessen am Gleichgewichtslohnsatz - zu hohe Lohnansprüche begründet ist.

Auf dieser Überlegung gründen sich denn auch alle wirtschaftspolitischen Ansätze, Arbeitslosigkeit durch Lohnmoderation zu bekämpfen. In diesem Kontext sind zudem alle Bemühungen zu sehen, Arbeitslosigkeit durch niedrige Lohnnebenkosten und möglichst schwache Gewerkschaften zu bekämpfen, um deren Lohnforderungen zu mäßigen. Das Marktsystem - durch diese Brille gesehen - liefert nicht nur zu jedem Zeitpunkt optimale Ergebnisse; es ist auch in sich stabil. Dies ist an die Voraussetzung geknüpft, dass es keinerlei störende Einflüsse von „außen“ gibt. Außen ist dabei auch der Staat. Der Wirtschaftspolitik kommt in dieser Denkschule allein die Rolle zu, die private Wirtschaftstätigkeit möglichst wenig zu stören und sie von Störungen frei zu halten. Genau deshalb sind eine an Regeln gebundene Geld- und Fiskalpolitik optimal. Sie geben den privaten Markteilnehmern einen verlässlichen Rahmen, innerhalb dessen diese ihre Entscheidungen fällen können. Aber auch jedwede andere Störung von außen verarbeitet dieses gedachte Markt- 
system scheinbar mühelos. Dies liegt daran, dass Unsicherheit in diesen Modellen in Gestalt bekannter Zufallsverteilungen vorkommt. Dieses Risiko können die privaten Marktteilnehmer gut bewältigen, weil sie qua Annahme über rationale Erwartungen verfügen. Diese Art der Erwartungsbildung impliziert, dass allen Marktteilnehmern das grundlegende Modell der Volkswirtschaft bekannt ist; sie wissen also, wie die Wirtschaft funktioniert und bilden auf dieser Basis ihre Erwartungen über die unsichere Zukunft. Darüber hinaus kennen sie auch noch die Wahrscheinlichkeitsverteilung des Risikos. Ausgestattet mit diesem profunden Wissen treffen sie systematisch richtige Entscheidungen. Anders formuliert: Unsichere Ereignisse überraschen sie nicht systematisch, sondern nur im Einzelfall. Das gilt auch für wirtschaftspolitische Maßnahmen. Diese verlieren sogar ihre Wirksamkeit, wenn sie in systematischer Weise auf der Überraschheit der Privaten basieren. Das schließt zum Beispiel eine Geldpolitik aus, die mit immer wieder überraschend hohen Inflationsraten zu unerwartet niedrigen Reallöhnen und Realzinsen führt, durch die die Wirtschaft stimuliert werden soll. Die Marktteilnehmer lassen sich aber unter diesen Voraussetzungen nicht systematisch täuschen, so dass die Wirkung dieser Politik in immer höheren Inflationsraten verpufft.

Genau dieses Beispiel theoretischer Überlegungen in Verbindung mit empirischen Untersuchungen aus den siebziger und achtziger Jahren wurde seither immer wieder als Argument gegen stabilisierende geld- und fiskalpolitische Maßnahmen herangezogen. In den wissenschaftlichen makroökonomischen Debatten jener Jahre wurde das dargestellte Grundmodell noch in vielfältiger Weise - zum Beispiel durch eine Dynamisierung des Gleichgewichtsbegriffs - modifiziert, doch die Grundstruktur der Argumentation blieb bis zur Finanzmarktkrise erhalten. In den Jahren davor hatte sich sogar ein weitgehender Konsens mit den vorherrschenden keynesianischen Denkströmungen herausgebildet, der so genannte Washington Consensus. Gegenüber dem dargestellten Grundmodell unterscheidet sich dieser dadurch, dass wegen möglicher Informations- und Koordinationsprobleme von einer geringeren Flexibilität des Preis- und Lohnmechanismus ausgegangen wird. Dies hat zur Konsequenz, dass sich kurzfristig durchaus Gleichgewichte bilden können, in denen nicht alle gewünschten Transaktionen ausgeführt werden können. Mit anderen Worten: Kurzfristig ist im Rahmen dieser Ansätze das Auftreten unfreiwilliger Arbeitslosigkeit durchaus möglich. Daher lässt sich für diese Ansätze eine Rechtfertigung für eine primär geldpolitische Stabilisierungspolitik finden, die allerdings nur kurzfristig wirksam sein kann. Ansonsten ergeben sich - insbesondere auf längere Sicht - die gleichen Schlussfolgerungen wie im dargestellten Grundmodell. 


\section{Marktkonforme Wirtschaftspolitik}

Diese Modelle lieferten die theoretische Grundlage für eine Politik, die sich stark an den aus heutiger Sicht vermeintlichen Bedürfnissen des Marktes orientierte. Diese Bedürfnisse bestanden in einer möglichst von staatlichen Eingriffen unbehinderten Entwicklung. Das implizierte vor allem den Abbau von Regulierungen. Dies geschah so für die Güter- und Dienstleistungsmärkte, aber auch für den Arbeitsund den Finanzmarkt. In den USA und Großbritannien setze diese Entwicklung Ende der siebziger und Anfang der achtziger Jahre unter der US- Präsidentschaft von Ronald Reagan bzw. der britischen Regierung von Margaret Thatcher teilweise mit massiver Wucht ein. In Deutschland vollzog sich dieser Prozess unter der Regierung von Bundeskanzler Kohl wesentlich zögerlicher, was von den Anhängern dieser Politik immer wieder als Reformstau kritisiert wurde. Erst mit der rot-grünen Regierung unter Bundeskanzler Schröder gewann der so bezeichnete Reformprozess an Fahrt. Insbesondere durchgreifende Deregulierungen des Arbeits- und des Finanzmarktes fanden in dieser Zeit statt. Dies geschah alles in der durch die gängige makroökonomische Theorie genährten Hoffnung, dass von staatlichen Eingriffen unbehelligte private Entscheidungen letztendlich auch zum gesamtwirtschaftlich optimalen Ergebnis führen. Der Kapitalismus, beruhend auf privater Verfügungsgewalt, wurde als in sich stabil angesehen. Deshalb erschien es auch in der deutschen Wirtschaftspolitik richtig, über die Deregulierung insbesondere im Niedriglohnbereich soziale Risiken wie Arbeitslosigkeit, Krankheit und Alter auf den Einzelnen zu verlagern. Auf diese Weise wurden Anreize zur Eigenvorsorge und für stärkere individuelle Anstrengungen gesetzt. Mit der gleichen Logik wurden auch die Güterund Finanzmärkte dereguliert. Hinter diesen Maßnahmen stand immer die Hoffnung auf ein beschleunigtes Wachstum und eine höhere Beschäftigung.

Ein Desiderat dieser ökonomischen Sichtweise ist das Bild von Staaten im Standortwettbewerb. Dies ist nichts anderes als die Anwendung der Konzepte unternehmerischen Wettbewerbs auf Staaten. Demnach sollten Staaten ihr Steuer- und Sozialsystem auf der einen und ihre Infrastruktur auf der anderen Seite so gestalten, dass sie attraktiv für Unternehmen sind. Diese würden sich dann dort ansiedeln bzw. an diesem Standort verbleiben. Auch auf diese Weise würde ein höheres Wachstum mit höherer Beschäftigung garantiert. Das Bild von Staaten im Standortwettbewerb hat die Wirtschaftspolitik in Deutschland während des vergangenen Jahrzehnts geprägt. Es lieferte die Begründung für Kürzungen im Sozialbereich, die vor allem niedrigere Abgabensätze für Unternehmen zur Folge haben sollten. Es stand Pate bei der Aufgabe des synthetischen Einkommenssteuermodells, das eine gleich hohe Besteuerung aller Einkommensarten vorsah. Stattdessen wurde über eine Absen- 
kung des Steuersatzes für Unternehmenssteuer, Einkommen aus Unternehmertätigkeit und Vermögen steuerlich privilegiert. Dies geschah in einem Ausmaß, dass der Staatshaushalt defizitär und die Infrastrukturmaßnahmen eher gekürzt wurden. Außerdem wurden die Verbrauchssteuern erhöht, die primär von den privaten Haushalten getragen werden.

Im Ergebnis ergab sich eine massive Umverteilung zugunsten der Unternehmensund zulasten der Arbeitseinkommen. In der Verteilung nahm die Ungleichheit der Einkommen und Vermögen in Deutschland auch im internationalen Vergleich erheblich zu. Schließlich gehören die Einkommen aus Unternehmertätigkeit und Vermögen in der Regel zu den höheren Einkommensgruppen. Diese waren Profiteure einer solchen Politik. Eine größere Ungleichheit wurde aber durch die Brille der vorherrschenden Theorien gleichfalls als eine Voraussetzung für eine höhere Beschäftigung gesehen, denn niedrigere Löhne am unteren Ende der Einkommensskala sollten die Einstellung niedrig produktiver Arbeitskräfte erleichtern. Dagegen sollten höhere hohe Einkommen die Anreize für Arbeitsanstrengungen verstärken.

\section{Eine Kritik der naiven Marktkonformität}

Die wirtschaftspolitische Perspektive der jüngeren Vergangenheit war von Grund auf verfehlt. Die markantesten Beweise für diese These sind die Finanzmarktkrise und ihre Folgekrise, die Krise des Euroraums, denn diese haben ihren Ursprung in genau jenen wirtschaftspolitischen Maßnahmen, die zuvor als unerlässliche Voraussetzungen für ein hohes Wachstum und eine hohe Beschäftigung gesehen wurden.

In erster Linie ist die Deregulierung der Finanzmärkte zu nennen. Sie hat zu einer Übernahme von Risiken geführt, die letztlich nicht mehr beherrschbar waren und nicht nur den gesamten Finanzsektor, sondern die gesamte Weltwirtschaft in eine tiefe Rezession gezogen haben, von der sich die meisten Volkswirtschaften auch fast fünf Jahre später noch nicht erholt hatten. Statt des versprochenen hohen Wachstums und hoher Beschäftigung ist in den meisten Ländern das Gegenteil eingetreten. In zweiter Linie ist auch die teilweise dramatisch erhöhte Ungleichheit von Einkommen und Vermögen eine der Quellen für die Krisen. Die Zusammenballung von Reichtum führt zu einem breiteren Strom Anlage suchenden Kapitals, dem auf den Finanzmärkten mit entsprechenden, teilweise sehr risikoreichen Angeboten Rechnung getragen wird. Nur mit diesem Strom in Kombination mit deregulierten Finanzmärkten war die Aufblähung des Finanzsektors überhaupt möglich. Ungleichheit erhöht somit die Anfälligkeit für Stabilitätsrisiken. Schließlich ist noch der Standortwettbewerb zu nennen, der im globalen Maßstab zu Handelsungleich- 
gewichten geführt hat, was in Gegenwart von Wechselkursen schlimmstenfalls unangenehme, aber relativ schmerzfrei überwindbare Währungsturbulenzen zur Folge hat. Innerhalb von Nationalstaaten wird solchen Ungleichgewichten durch unterschiedliche Kompensationsmechanismen präventiv Rechnung getragen. Schon eine nationale Arbeitslosenversicherung trägt zur Stabilisierung bei, erst recht solche komplexen Verfahren wie der Länderfinanzausgleich in Deutschland. Innerhalb des Euroraums, in dem es weder Wechselkurse noch Kompensationsmechanismen gibt, zeigt ein Standortwettbewerb dagegen verheerende Konsequenzen. Die erfolgreichen Länder häufen Überschüsse im Außenhandel an. Mit anderen Worten: Sie erhöhen ständig ihr Auslandsvermögen. Das mag auf den ersten Blick positiv und als Ausweis von wirtschaftlicher Stärke erscheinen. Man muss jedoch berücksichtigen, dass dem Vermögen der einen zwangsläufig die Verschuldung der anderen gegenübersteht. Wenn beides über lange Zeiträume steigt, kommt irgendwann der Zeitpunkt, an dem die Solvenz des Schuldners an den Finanzmärkten in Frage gestellt wird. Das ist der Zeitpunkt, an dem Anleger ihr Geld abziehen und das Vermögen in der Regel drastisch an Wert verliert. Anders ausgedrückt, die Erfolge des Standortwettbewerbs verflüchtigen sich.

Die Mühen waren vergeblich. An dieser Stelle fällt die Analogie zum unternehmerischen Wettbewerb in sich zusammen. Ein Unternehmen, das im Wettbewerb nicht besteht, scheidet aus dem Markt aus. Das ist für die Eigentümer und Beschäftigten sehr schmerzhaft, für die Gesamtwirtschaft ist es jedoch vorteilhaft. Die Konkurrenz, die offensichtlich bessere Produkte anbietet, profitiert vom Ausscheiden des Wettbewerbsverlierers. Sie kann ihre Gewinne und ihre Beschäftigung erhöhen, und für die Gesamtwirtschaft ergibt sich ein verbesserter Ressourceneinsatz und Produktmix. Ganz anders sieht es beim Wettbewerb zwischen Staaten aus. Ein Staat, der insolvent ist, scheidet nicht aus dem Markt aus. Er besteht fort. Eine staatliche Zahlungsunfähigkeit ist nicht nur für die Regierungen und im öffentlichen Dienst Beschäftigten schmerzhaft, sondern auch für die Gesamtwirtschaft. Ein Staat verschwindet nämlich nicht vom Markt und wird von einem besseren ersetzt, ebenso wenig wie seine Bürger. Auch die anderen Staaten profitieren nicht von seiner Pleite. Im Gegenteil, in den Gläubigerländern müssen Vermögen abgeschrieben werden. Auch das Angebot an Gütern verbessert sich nicht. Wiederum ist das Gegenteil der Fall, ein insolventer Staat wird sein Angebot an öffentlichen Gütern nicht aufrechterhalten können und damit auch noch den Rest der Wirtschaft schwer schädigen. Das Fazit aus alledem lautet: Die Übertragung privatwirtschaftlicher Wettbewerbskonzepte auf den Staat ist dysfunktional. Sie führt nicht zu mehr Wachstum und mehr Beschäftigung, sondern zum Gegenteil. 
Anhand dieser Überlegungen lässt sich pars pro toto der grundlegende ökonomische Denkfehler aufzeigen, der die jüngere Wirtschaftspolitik geprägt hat. Es ist der Glaube an die generelle Überlegenheit einzelwirtschaftlicher, privater Entscheidungen in Kombination mit der Unfähigkeit, die destabilisierenden systemischen Folgen solcher Entscheidungen richtig einzuschätzen. Der Kapitalismus ist eben nicht inhärent stabil. Setzt man dies aber voraus und überdehnt in diesem Glauben gleichsam die Anwendung privater Verfügungsgewalt, erntet man Krisen in verschiedenster Ausprägung: Eine Finanzmarktkrise, die aus dem Vertrauensverlust in die Stabilität eines deregulierten Finanzsystems resultiert, und eine Krise des Euroraums, weil die Anleger kein Vertrauen mehr in dessen unzureichendes institutionelles Gefüge haben, das den staatlichen Standortwettbewerb ungebremst zur Entfaltung kommen lässt - mit all seinen destabilisierenden Wirkungen auf die in diesem Wettbewerb scheiternden Staaten. Es war also das einzelwirtschaftliche Denken der Kapitalisten und dessen Übernahme durch die Wirtschaftspolitik, die den Kapitalismus in die Krise geführt haben. Die Krise des Kapitalismus - die insbesondere Europa beginnend seit 2007 (und seit 2009 verschärfend der Euroraum) gerade durchlebt - ist also das Ergebnis seiner Ideologisierung. Die uneingeschränkte Anwendung kapitalistischer Prinzipien zerstört die Grundlagen des Kapitalismus und erzeugt Krisen. Das sollten vor allem jene bedenken, die in einer marktkonformen Demokratie eine erstrebenswerte wirtschaftspolitische Option sehen.

\section{Die Option eines beschränkten Kapitalismus}

Es gilt jetzt Erkenntnisse aus dem vergangenen Jahrhundert aufzufrischen. Schon einmal, nach der großen Depression in den dreißiger Jahren, stand man vor den Trümmern des kapitalistischen Systems. Auch damals hatte man dessen inhärente Stabilität maßlos überschätzt. Als Konsequenz suchten viele ihr Heil in einem Staatssozialismus, den man als stabiler ansah. Dies entpuppte sich in Verbindung mit dessen mangelnder wirtschaftlicher Dynamik gleichfalls als Illusion und kann mittlerweile als gescheitert betrachtet werden. Daneben entstanden aber Konzepte eines gezähmten Kapitalismus. Diese Konzepte reichten vom Ordoliberalismus bis hin zum Keynesianismus. All diesen Konzepten ist gemeinsam, dass sie die inhärente Instabilität des Kapitalismus kennen. Über deren Wurzeln besteht allerdings Uneinigkeit. Während Ordoliberale die inhärente Gefährdung des Wettbewerbs betonen, sehen Keynesianer die eigentliche Gefahr in der fundamentalen Unsicherheit wirtschaftlichen Handelns und dessen Folgen für die gesamtwirtschaftliche Nachfrage. Ersteres wird in den modernen makroökonomischen Modellen in Zeiten har- 
ten globalen Wettbewerbs als nicht mehr relevant angesehen, Letzteres wird zumeist als Risiko verharmlost, dessen Wahrscheinlichkeitsverteilung in der Regel sogar allen Akteuren bekannt ist. Diese Naivität im Umgang mit Marktsystemen ist nicht mehr aufrechtzuerhalten. Leider wird dies von der gegenwärtigen Politik, die immer zu stark auf einzelwirtschaftlichen Argumentationsketten basiert, zumeist übersehen. Es bedarf Beschränkungen privaten Handelns, um dessen gesamtwirtschaftliche Stabilität zu gewährleisten.

Solche Schranken, die nach der Großen Depression insbesondere für die Aktivität auf Finanzmärkten errichtet und die im letzten Viertel des 20. Jahrhunderts zunehmend als Wachstumshemmnisse gesehen wurden, müssen in modernisierter Form wieder errichtet werden. Denn nur ein Kapitalismus, der nicht von einer kapitalistisch denkenden und handelnden Wirtschaftspolitik begleitet wird, wird Bestand haben.

Korrespondenzanschrift:

Prof. Dr. Gustav A. Horn

Institut für Makroökonomie und Konjunkturforschung

Hans-Böckler-Straße 39

40476 Düsseldorf

E-Mail: Gustav-Horn@boeckler.de 